\title{
Quality Analysis and High-Performance Liquid Chromatographic Fingerprint Analysis of New Cultivated Kind of Lonicerae japonicae Flos "Hua Jin 6" from Different Harvest Times
}

\author{
Lingna Wang, Yongqing Zhang \\ School of Pharmacy, Shandong University of Traditional Chinese Medicine, Jinan, China \\ Email: zyq622003@126.com
}

How to cite this paper: Wang, L.N. and Zhang, Y.Q. (2017) Quality Analysis and High-Performance Liquid Chromatographic Fingerprint Analysis of New Cultivated Kind of Lonicerae japonicae Flos "Hua Jin 6" from Different Harvest Times. Chinese Medicine, 8, 18-32.

https://doi.org/10.4236/cm.2017.81003

Received: January 11, 2017

Accepted: March 24, 2017

Published: March 27, 2017

Copyright $\odot 2017$ by authors and Scientific Research Publishing Inc. This work is licensed under the Creative Commons Attribution International License (CC BY 4.0).

http://creativecommons.org/licenses/by/4.0/

\begin{abstract}
Lonicerae japonicae Flos (LJF) is widely used in traditional Chinese medicines for the treatment of various diseases, which is now in great demand every year and has a broad development prospect. However, the flowering phase of common LJF varieties is so short, which seriously restricts the development of LJF industry. As a new cultivated kind of Lonicerae japonicae Flos, "Hua Jin 6" has characteristics in long flowering phase and conveniently picking, which makes it have a broad development prospect. The aim of this study is to provide scientific guidance for its suitable harvest period by measuring yield and quality of "Hua Jin 6" from different harvest time. Studies show that flower size had a slowly rising trend from the first day to the seventh day, and then slowly declined or kept stable. There were no significant differences of total phenolic acid contents in different samples from different days, but contents of total flavonoids were on the rise and up to maximum in the ninth day. The contents of total iridoids had an increasing tendency from the first day to the fifth day and then kept relatively stable in other days. We demonstrated that the quality of "Hua Jin 6" is relatively stable and suitable for harvesting in all flower buds white stage in term of HPLC fingerprints. Our findings can make it possible to select the suitable time for different harvest purpose.
\end{abstract}

\section{Keywords}

Lonicerae japonicae Flos, New Cultivate Kind, "Hua Jin 6", Quality Analysis, HPLC Fingerprint, Harvest Time

\section{Introduction}

Lonicerae japonicae Flos (LJF), the dried flower buds of Lonicera japonica 
thunb., is well known and commonly used as herbal medicine for treatment of various diseases in traditional Chinese medicine (TCM) practice, such as antiinflammatory, anti-bacterial, anti-viral, anti-oxidative and anti-diabetic activities [1] [2] [3] [4] [5]. Generally, the flowering stage of LJF has been divided into three stages: the green bud stage (Bud size $1.0-3.5 \mathrm{~cm}$, green), the white bud stage (Bud size $3.0-4.5 \mathrm{~cm}$, white), the flowering stage (Blooms) [6]. Research has shown that the white bud stage is the most appropriate harvest time of LJF according to the maximum yield and superior quality [7]. However, the lasting time from the white bud stage to the flowering stage is so short (2 - 3 days) and the opening buds are not up to official standards. Therefore, a lot of manpower are needed to pick flower buds in the period of flower phase and numerous wealth are consumed. As a result, many farmers have abandoned cultivating or collecting LJF and a great deal of economic losses [8] [9].

"Hua Jin 6" is a new Lonicerae japonicae Flos variety selected in ten years from natural hybrid progeny of local variety, and the most prominent characteristics of the variety is the white bud stage which can last up to 15 days then to open or directly wither and fall. Furthermore, the high output is another feature of "Hua Jin 6" because the flower buds are much denser and bigger than common varieties. As a result, white flower buds can be a one-time picking if farmers cultivate "Hua Jin 6" which could slash personnel costs and improve economic returns. Besides, previous studies showed that the output of "Hua Jin 6" was 1.64 times higher than that of "Hua Jin 2"; contents of chlorogenic acid and cynaroside were respectively 2.03 times and 2.8 times higher than those of "Hua Jin 2" [9]. Therefore, its potential applications are fantastic from above mentioned characteristics. However, whether it is suit to pick during all white flower bud stage or not depends on the flowering quality change laws, so it is the most important task to study on dynamic changes of "Hua Jin 6" quality and yield during all white flower bud stage to determine the suitable harvest time.

Generally, the suitable harvest time of herbal medicines is determined according to the maximal yield and the best quality. Similarly, the best harvest time of "Hua Jin 6" also depend on yield and quality. Usually, yield refers to flower buds weight and size, but there are not unity standard index to evaluate the quality of LJF. In the Chinese Pharmacopoeia (2015 version), only two components (chlorogenic and cynaroside) were used to evaluate the quality of LJF products. While, we all known that for the herbal medicines the safety and efficiency are not just attributed to one or several particular constituents, it usually depends on the interactions among these constitutes which can make their relationship to the safety and efficacy much more complicated than that pure components [10] [11]. Therefore, we should take into account as many as possible constitutes to evaluate the quality of the herbal medicines. Besides, chromatographic fingerprint using high-performance liquid chromatography (HPLC), which focuses on the systemic characterization of composition of the complex chemical mixture, is becoming increasingly recognized as an important separation technique. Recently, several studies on the chromatographic fingerprinting using HPLC tech- 
nique have been reported [12] [13] [14].

In this study, we determined morphological characteristics (bud length and bud diameter) and buds dried weight of "Hua Jin 6" from the first day to the eleventh day of the white bud stages to evaluate the yield. Meanwhile, a rapid and simple HPLC method for quantitative determination of 11 major active components from LJF was established, which included 6 phenolic acids, 2 flavonoids and 3 iridoids, the chemical structures of 11 components are shown in Figure 1. Finally, chromatographic fingerprint analysis with HPLC was developed to evaluate the stability of "Hua Jin 6" from different harvest time.

\section{Experimental}

\subsection{Plant Material}

Samples of "Hua Jin 6" from different harvest times were collected in medicinal botanical garden of Shan Dong University of TCM of China and identified by<smiles>CC(=O)/C=C/c1ccc(O)c(O)c1</smiles>

Chlorogenic Acid (CA) $\mathrm{R}_{1}=$ caffeoyl $\mathrm{R}_{2}=\mathrm{R}_{3}=\mathrm{H}$

Cryptochlorogenic Acid (CCA) $\mathrm{R}_{2}=$ caffeoyl $\mathrm{R}_{1}=\mathrm{R}_{3}=\mathrm{H}$

Neochlorogenic Acid (NCA) $\mathrm{R}_{3}=$ caffeoyl $\mathrm{R}_{1}=\mathrm{R}_{2}=\mathrm{H}$

Isochlorogenic Acid $B$ (ICA-B) $\mathrm{R}_{1}=\mathrm{R}_{2}=$ caffeoyl $\mathrm{R}_{3}=\mathrm{H}$

Isochlorogenic Acid A (ICA-A) $\mathrm{R}_{1}=\mathrm{R}_{3}=$ caffeoyl $\mathrm{R}_{2}=\mathrm{H}$

Isochlorogenic Acid C (ICA-C) $\mathrm{R}_{2}=\mathrm{R}_{3}=$ caffeoyl $\mathrm{R}_{1}=\mathrm{H}$<smiles>[R]c1cc(O)c2c(=O)c([R3])c(-c3ccc(O)c(O)c3)oc2c1</smiles>

Rutin (RT) $\mathrm{R}_{1}=\mathrm{O}$-glc-rha $\mathrm{R}_{2}=\mathrm{OH}$

Cynaroside $(\mathrm{CN}) \mathrm{R}_{1}=\mathrm{H} \mathrm{R}_{2}=\mathrm{O}$-glc

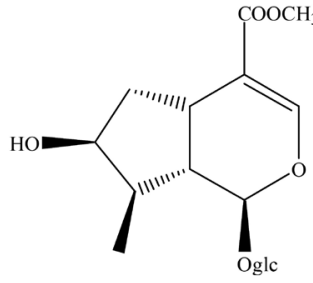

loganin (LG)

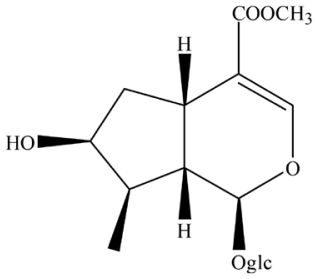

loganic acid (LGA)<smiles>C=C[C@H]1[C@@H](CC=O)C(C(=O)OC)=CO[C@H]1C(C)=O</smiles>

Secoxylogain ( SCLG)

Figure 1. Chemical structures of 11 active components. 
Prof. Zhang Yongqing from Shandong University of TCM. White flower buds of "Hua Jin 6" were picking from the first day to the eleventh day of the white bud stage and all samples were stored in Silica gel box to dry. Voucher specimens were preserved at herbarium of Shandong university of TCM (SDCM).

\subsection{Chemicals and Solvents}

The standard samples of RT was purchased from The National Institute For the Control of Pharmaceutical and Biological Products, NCA, CA, CCA, ICA-B, ICA-A, ICA-C, LG, CN, HP, LGA were purchased from Shanghai Yuanye BioTechnology Co., Ltd. SCLG was purchased from Shanghai Guyan Bio-Technology Co., Ltd. The purities of above mentioned chemical components were all $\geq$ 98\%, and their structures are shown in Figure 1.

Acetonitrile and formic acid of HPLC grade were chromatographically pure and other reagents were analytically pure. The double distilled water was selfmade. Analytical grade methanol was purchased from Tianjin Fuyu Fine Chemical Co., Ltd. and used for sample preparation.

\subsection{Flower Bud Sizes and Dried Weight}

Flower bud sizes: Samples from different harvest times were randomly divided into 10 groups, each groups included 10 dried buds, we determined bud length and bud diameter using ruler $(0.1 \mathrm{~mm})$ and vernier caliper $(0.01 \mathrm{~mm})$ respectively, and take an average.

Flower dried weight: Samples from different harvest times were randomly divided into 10 groups, each groups included 10 dried buds, we determined flower dried weight of each group using electronic balance and then take an average.

\subsection{HPLC Analysis [10]}

The analysis were performed on an Agilent 1260 liquid chromatography system, equipped with a quaternary gradient pump, an autosampler a diode array detector; A ZORBAX SB-C18 $(4.6 \mathrm{~mm} \times 250 \mathrm{~mm}, 5 \mathrm{um})$ column at temperature of $25^{\circ} \mathrm{C}$ was applied for all analyses. Detection wavelengths were sat at $327 \mathrm{~nm}$ for phenolic acids (NCA, CA, CCA, ICA-B, ICA-A, ICA-C), $350 \mathrm{~nm}$ for flavonoids (RT, CN), $240 \mathrm{~nm}$ for iridoids (LG, LGA, SCLG). The mobile phase consisted of $0.2 \%$ aqueous formic acid (A) and acetonitrile (B) in a gradient elution mode was as follows: 0 - $10 \mathrm{~min}$ : $92 \%-85 \% \mathrm{~A}, 10-20 \mathrm{~min}$ : $85 \% \mathrm{~A}, 20-30 \mathrm{~min}: 75 \%$ A, 30 - 40 min: $75 \%$ - 55\% A, $40-60$ min: $55 \%$ - 0\% A. The flow rate was 1.0 $\mathrm{ml} / \mathrm{min}$ and aliquots of $20 \mathrm{uL}$ were injected. The HPLC peaks were identified by comparing the retention times and the UV absorption of the 11 active components in each sample with those of the standard solutions. HPLC chromatograms of samples and mixture reference substances are shown in Figure 2.

\subsection{Similarity Analysis}

On the basis of the HPLC data, the similarities of LJF samples were performed by a professional software named Similarity Evaluation System for Chromatographic 


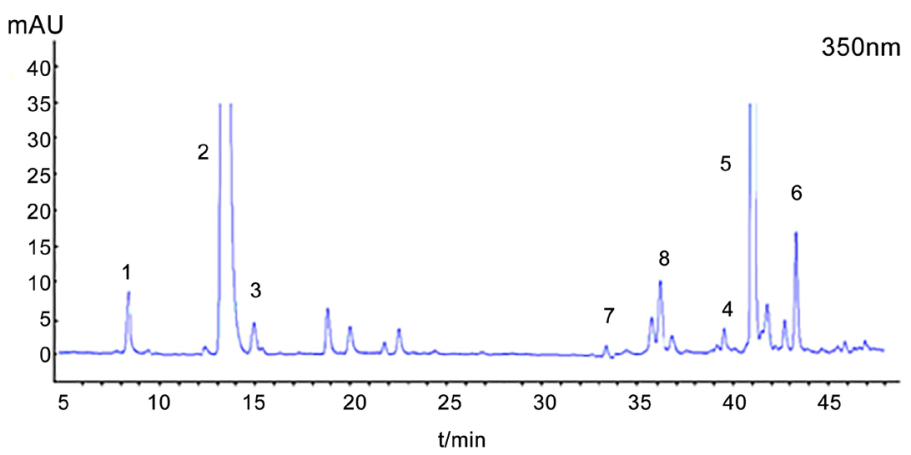

(a)

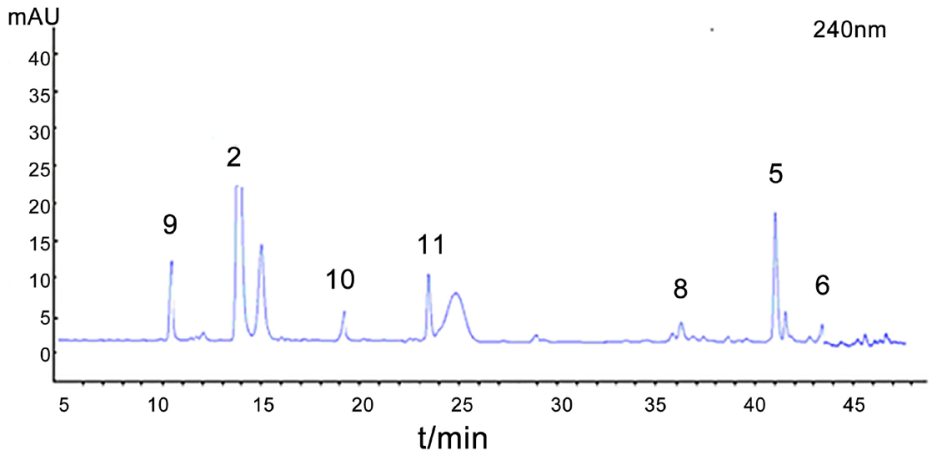

(b)

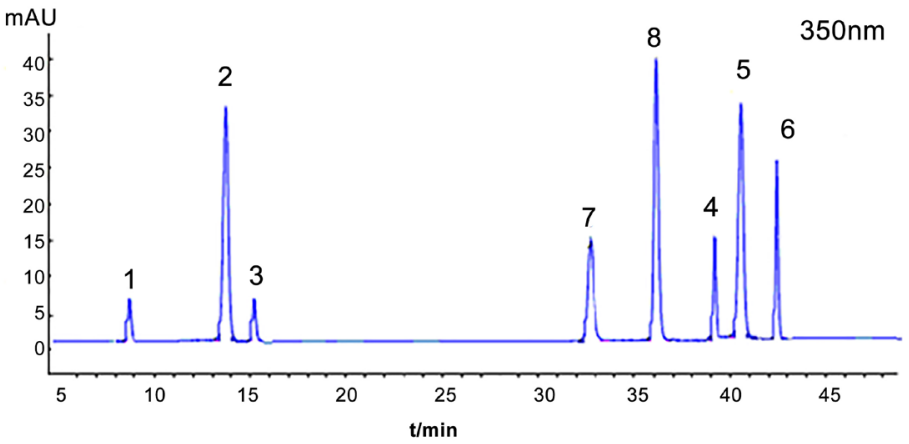

(c)

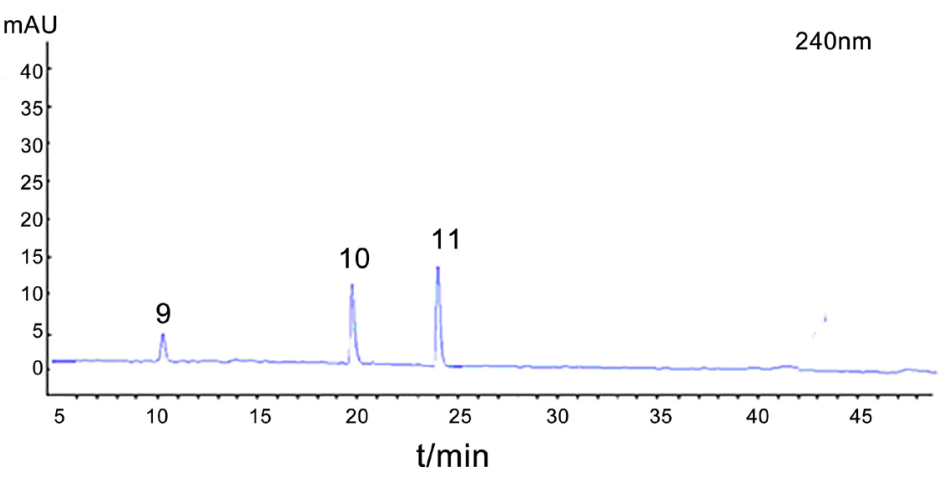

(d)

Figure 2. HPLC chromatogram of sample ((a) $350 \mathrm{~nm}$; (b) $240 \mathrm{~nm}$ ) and mixture reference substances (c) $350 \mathrm{~nm}$; (d) $240 \mathrm{~nm}$ ): (1) NCA; (2) CA; (3) CCA; (4) ICA-B; (5) ICA-A; (6) ICA-C; (7) RT; (8) CN; (9) LGA; (10) LG; (11) SCLG. 
Fingerprint (SESCF) of TCM (Version 2004 A) composed by Chinese Pharmacopoeia Committee, which was recommended by CFDA of China. Simultaneously, a reference chromatogram, including all the common peaks in the chromatograms of the 11 samples, was carried out by the median method, and the similarities between the reference chromatogram and the 11 samples were calculated.

\subsection{Statistical Analysis}

Statistical evaluation was carried out using one-way analysis of variance (ANOVA followed by Dunnett's post hoc test). Statistical differences were considered to be significant at $\mathrm{P}<0.05$. The programs were analyzed using the software named GraphPad Prism 5.

\section{Results}

\subsection{Validation of the HPLC Quantitative Method}

The calibration curves were constructed using external standard method. The contents of 11 analytes were the average values of three replicate injections. The corresponding regression equation and other characteristic parameters for the determination of analytes were shown in Table 1 . All calibration curves exhibited excellent linear behaviour $(\mathrm{R} 2>0.999)$ in a relatively wide concentration range. The LODs $(\mathrm{S} / \mathrm{N}=3)$ and LOQs $(\mathrm{S} / \mathrm{N}=10)$ for the analytes were less than 0.28 and $0.78 \mu \mathrm{g} / \mathrm{mL}$, respectively, indicating that the proposed HPLC method presented excellent sensitivity and was successfully applied for determination of 11 active components in LJF.

\subsection{Changes of Flower Bud Length and Diameter of LJF "Hua Jin 6" from Different Harvest Time}

Changes of flower bud length and diameter are shown in Figure 3. From the figure we can know that flower bud length and diameter had a slowly rising ten-

Table 1. Calibration curves, LODs and LOQs for 11 active components.

\begin{tabular}{cccccc}
\hline Analyte & Linearity range $(\mu \mathrm{g} / \mathrm{mL})$ & Calibration curve & $\mathrm{R}^{2}(\mathrm{n}=3)$ & LOD $(\mu \mathrm{g} / \mathrm{mL})$ & LOQ $(\mu \mathrm{g} / \mathrm{mL})$ \\
\hline NCA & $2.69-134.34$ & $\mathrm{y}=1822.3 \mathrm{x}+10.364$ & 0.9999 & 0.21 & 0.66 \\
CA & $2.73-1563.60$ & $\mathrm{y}=1851.3 \mathrm{x}+13.69$ & 0.9997 & 0.28 & 0.72 \\
CCA & $4.51-43.31$ & $\mathrm{y}=1432.7 \mathrm{x}+3.3910$ & 0.9995 & 0.18 & 0.62 \\
ICA-B & $3.70-27.73$ & $\mathrm{y}=2497.9 \mathrm{x}-9.6004$ & 0.9999 & 0.19 & 0.66 \\
ICA-A & $52.20-1257.10$ & $\mathrm{y}=2229.8 \mathrm{x}+27.950$ & 0.9999 & 0.26 & 0.72 \\
ICA-C & $13.08-303.30$ & $\mathrm{y}=1068.4 \mathrm{x}-4.9689$ & 0.9998 & 0.21 & 0.70 \\
RT & $1.926-441.11$ & $\mathrm{y}=591.67 \mathrm{x}-0.6221$ & 0.9998 & 0.23 & 0.71 \\
CN & $7.21-515.11$ & $\mathrm{y}=867.31 \mathrm{x}-4.3101$ & 0.9998 & 0.20 & 0.78 \\
LG & $11.37-30.32$ & $\mathrm{y}=1406.7 \mathrm{x}-2.0102$ & 0.9996 & 0.17 & 0.71 \\
LGA & $73.25-136.50$ & $\mathrm{y}=0.00125 \mathrm{x}+0.00799$ & 0.9998 & 0.24 & 0.73 \\
SCLG & $39.50-158.00$ & $\mathrm{y}=1374.4 \mathrm{x}+29.571$ & 0.9998 & 0.24 & 0.71 \\
\hline
\end{tabular}


dency and then had a slight reduce from the first day to the eleventh day. Among of them, flower bud diameter slowly increased from $0.2466 \mathrm{~cm}$ to 0.3424 $\mathrm{cm}$ during the first day to the tenth day, and then had a slightly reduce to 0.3272 $\mathrm{cm}$ in the eleventh day; flower bud length also had a minor increase from 2.8030 $\mathrm{cm}$ to $3.4336 \mathrm{~cm}$ from the first day to the ninth day, and then had a slight reduce to $3.0300 \mathrm{~cm}$ in the eleventh day. The result showed that the flower buds were also in the stage of vigorous growth during the first day to the tenth day and in the period of decline from the eleventh day.

\subsection{Changes of Flower Bud Dried Weight of LJF Hua Jin 6 from Different Harvest Time}

To a certain degree, flower bud dried weight has always been the main index limiting LJF yield. Changes of flower bud dried weight are shown in Figure 4, from the figure we can see clearly that flower bud dried weight was relatively

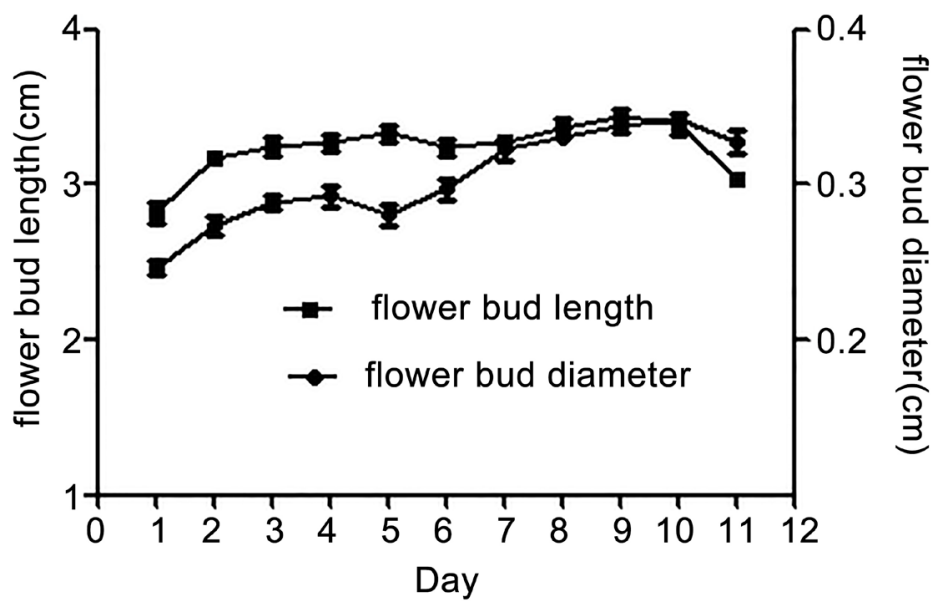

Figure 3. Changes of flower bud length and diameter $(\mathrm{cm})$ of LJF "Hua Jin 6 " from different harvest time.

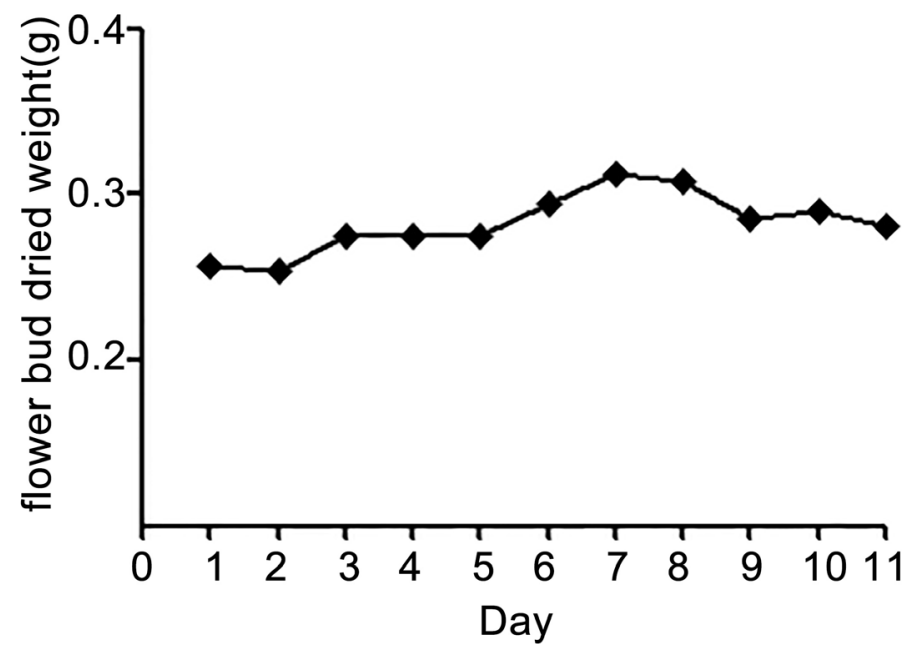

Figure 4. Changes of bud dry weight (g/10 buds) of LJF "Hua Jin 6" from different harvest time. 
stable during all white bud stage. Flower bud dried weight was slightly rising from $0.2577 \mathrm{~g}$ to $0.3129 \mathrm{~g}$ during the first day to the seventh day, and then had a slight reduce to $0.2905 \mathrm{~g}$ in eleventh day. From the result we can know that the accumulated amount of dry matter of LJF reached maximum in the seventh day and then slightly reduced or kept stable in another days. Therefore, we assumed the yield of LJF was much higher after the seventh day harvesting.

\subsection{Contents of Active Constituent of LJF “Hua Jin 6" from Different Harvest Time}

\subsubsection{Phenolic Acid Contents Analysis}

Contents of active constituent of LJF Hua Jin 6 from different harvest time are shown in Figure 5. From the figure we can see that except chlorogenic, the contents of other compounds were increase slowly as a whole, and the content of chlorogenic acid slowly reduces during the whole white flower bud stages from the first day to the eleventh day during white flower bud stages.

We also calculated the total contents of phenolic acids during the all flower buds white stage. From the Figure 5, we can know that there were no significant different of phenolic acid contents in different sample from different days (Figure 5). We speculated that chlorogenic acid synthesized into other phenolic acids with the growth of LJF. Therefore, the total phenolic acids contents were relatively stable in all flower buds white stage. The highest phenolic acid contents were detected in sample from the second day $(33.9890 \mathrm{mg} / \mathrm{g})$, while flower buds from the eighth day had the lowest content $(27.2338 \mathrm{mg} / \mathrm{g})$.

\subsubsection{Flavonoid Contents Analysis}

Changes of flavonoid contents of LJF "Hua Jin 6" from different harvest time are shown in Figure 6. From the figure we can clearly see that there were significantly different in flavonoid constituents. The variation law of rutin was not obvious. The content of cynaroside slowly increased in all days from $0.9444 \mathrm{mg} / \mathrm{g}$ to $1.9257 \mathrm{mg} / \mathrm{g}$.

It was also shown that there were significant different of the total flavonoid contents in different sample from different days (Figure 6), it had a increasing tendency from the first day to the eleventh day. The lowest flavonoid contents were detected in sample from the first day $(1.0340 \mathrm{mg} / \mathrm{g})$, while in the ninth day of flower bud white stages, the total flavonoids contents were nearly 2 times over those of the first day $(2.0251 \mathrm{mg} / \mathrm{g})$.

\subsubsection{Iridoid Contents Analysis}

Changes of iridoid contents of LJF Hua Jin 6 from different harvest time are shown in Figure 7. From the figure we can know that three iridoids had a slowly increase tendency and then reduced or kept relatively stable. Similarly, we calculated the total iridoid contents shown in Figure 7, form the figure we can know that there was an increasing tendency from the first day to the fifth day and then keep the relatively stable in other days.

By measuring 11 active components contents of LJF from different harvest 


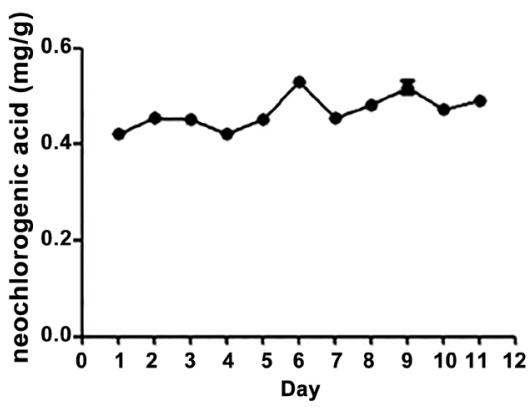

(a)

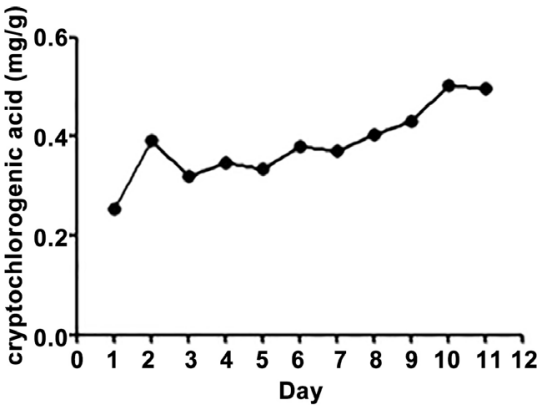

(c)

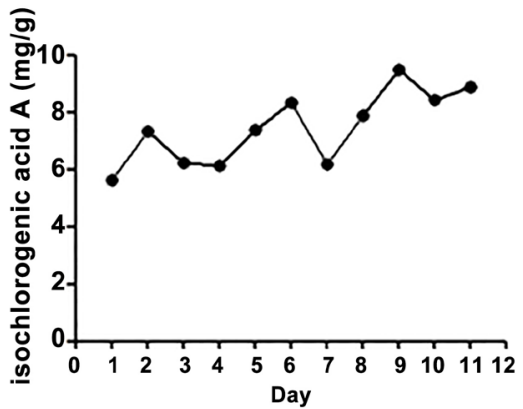

(e)

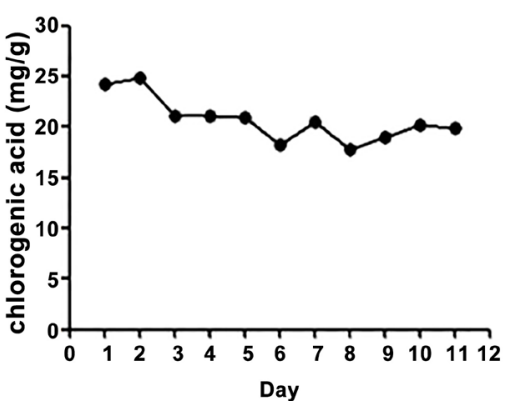

(b)

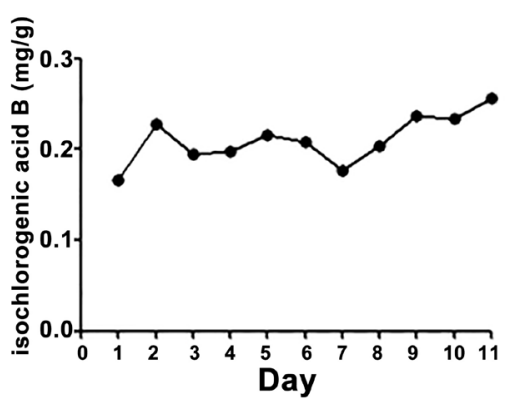

(d)

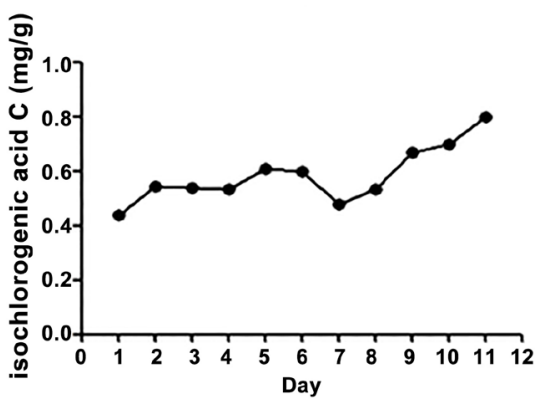

(f)

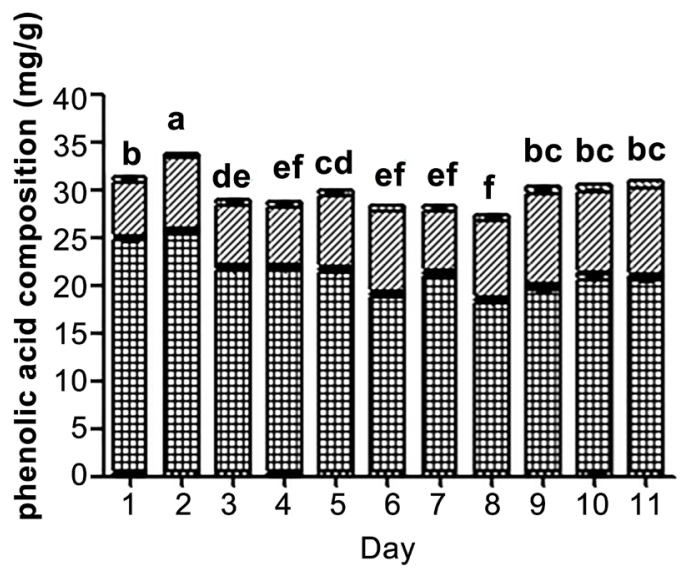

Figure 5. Changes of phenolic acid contents of LJF "Hua Jin 6" from different harvest time (a) NCA; (b) CA; (c) CCA; (d) ICA-B; (e) ICA-A; (f) ICA-C.

time, we known that the total phenolic acids contents were relatively stable in all bud white stages. However, the total flavonoids contents had an increasing tendency in all white bud stages, and flavonoids contents reached the highest in the 


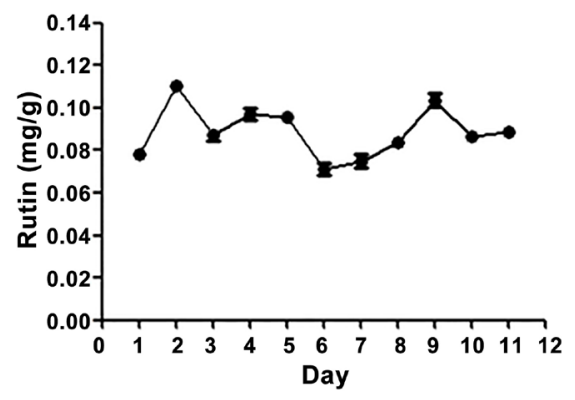

(a)

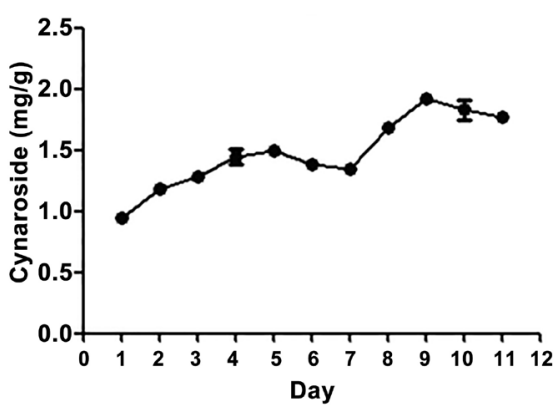

(b)

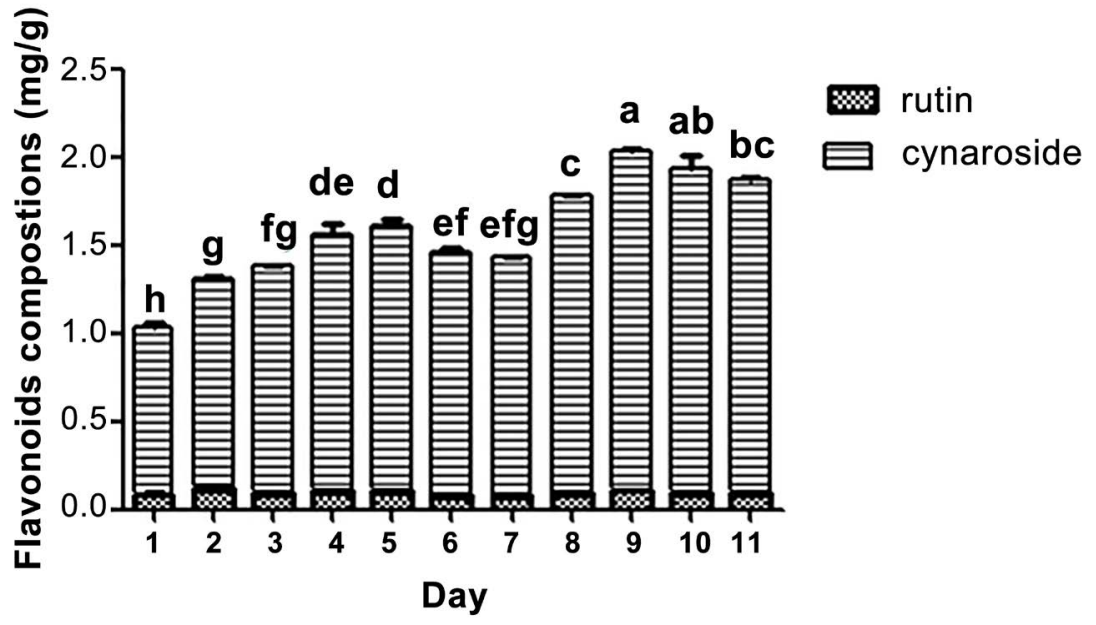

Figure 6. changes of flavonoid contents of LJF "Hua Jin 6" from different harvest time (a) $\mathrm{RT}$; (b) CN).

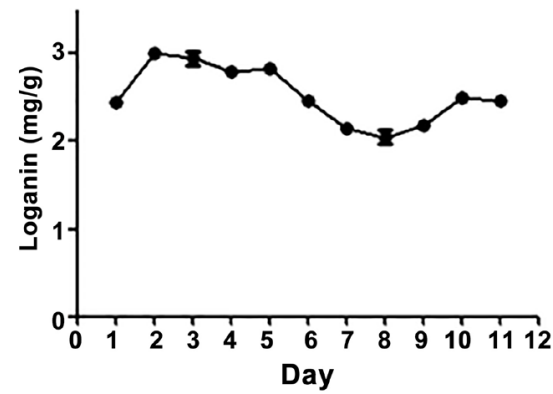

(a)

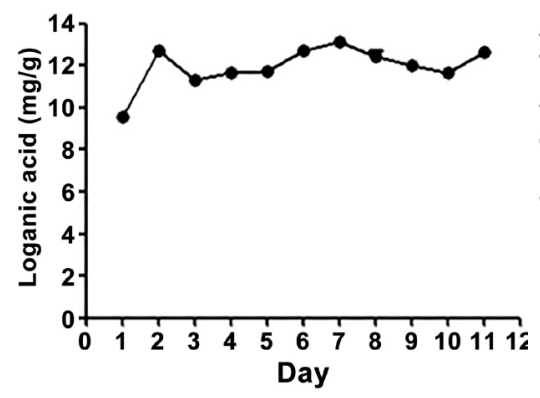

(b)

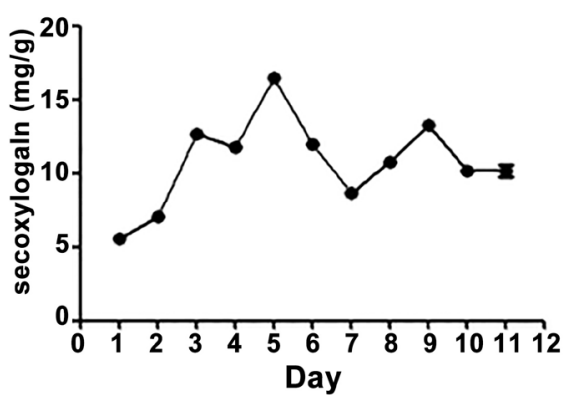

(c)

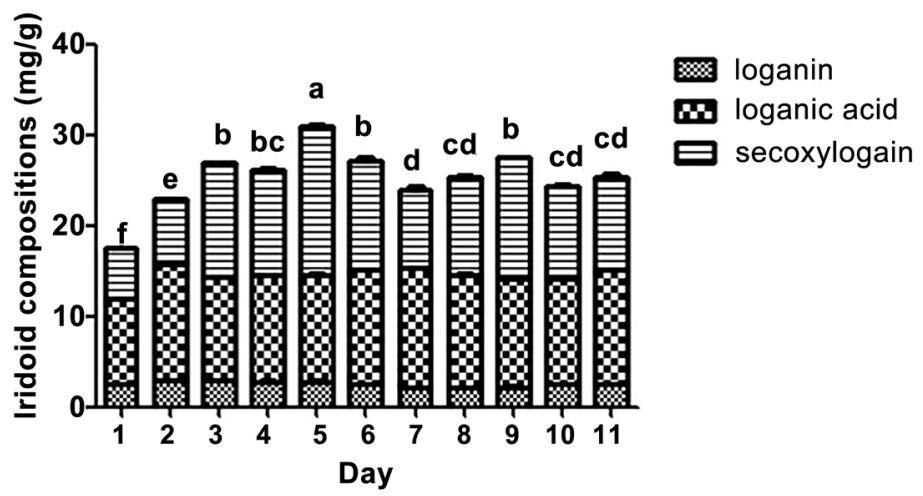

Figure 7. Changes of iridoid contents of LJF "Hua Jin 6" from different harvest time (a) LG; (b) LGA; (c) SCLG). 
ninth day, which had no remarkably significant with the eighth, the tenth, the eleventh day. Meanwhile, the total iridoid contents had an increasing tendency from the first day to the fifth day, then slowly declined in the following day. Taking above mentioned consideration, we can select appropriate harvesting time according different harvest purpose.

\subsection{HPLC Fingerprints of LJF}

\subsubsection{HPLC Fingerprint Analysis}

Based on the method of the samples determinated, HPLC fingerprints of LJF "Hua Jin 6" were obtained and are shown in Figure 8(a), which shown that 11

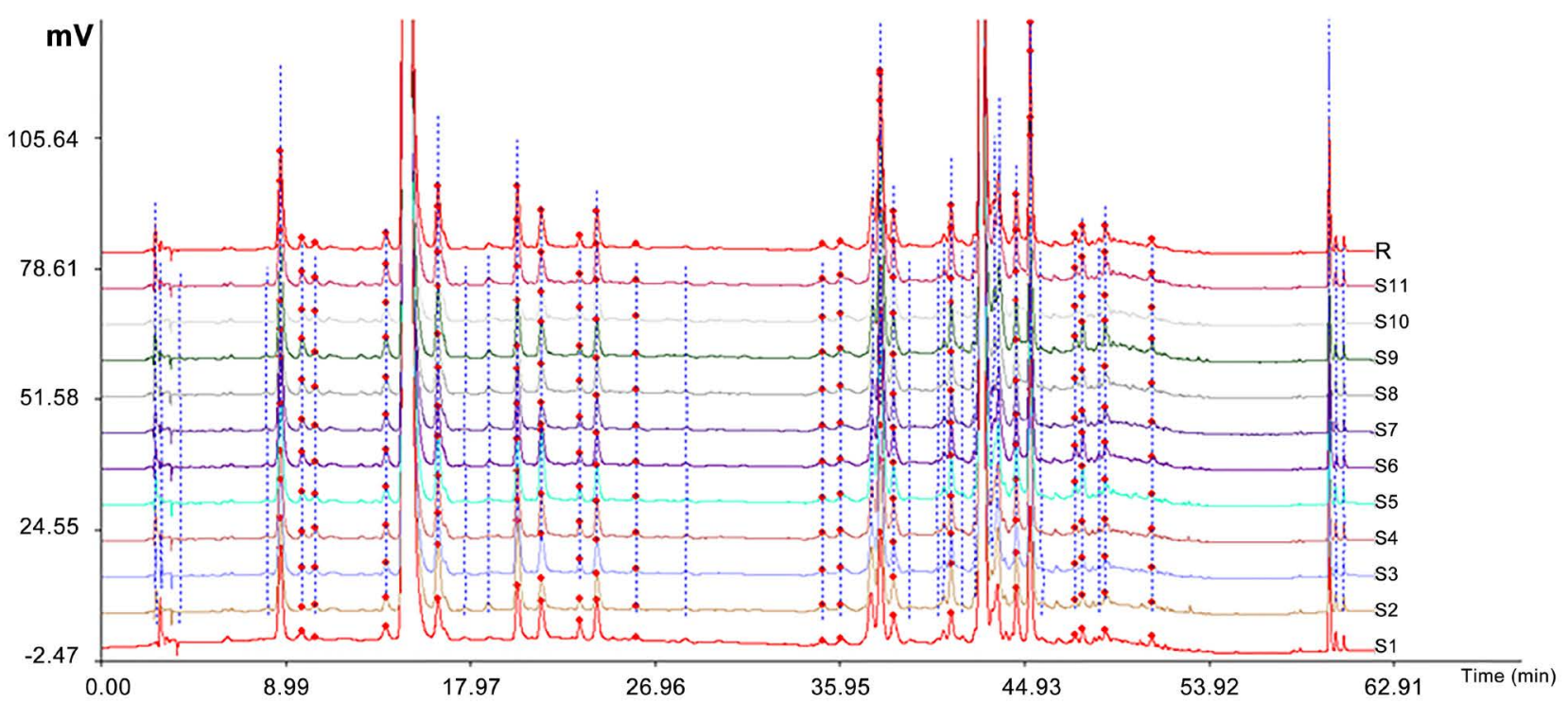

(a) Retention time ( $\mathrm{min})$

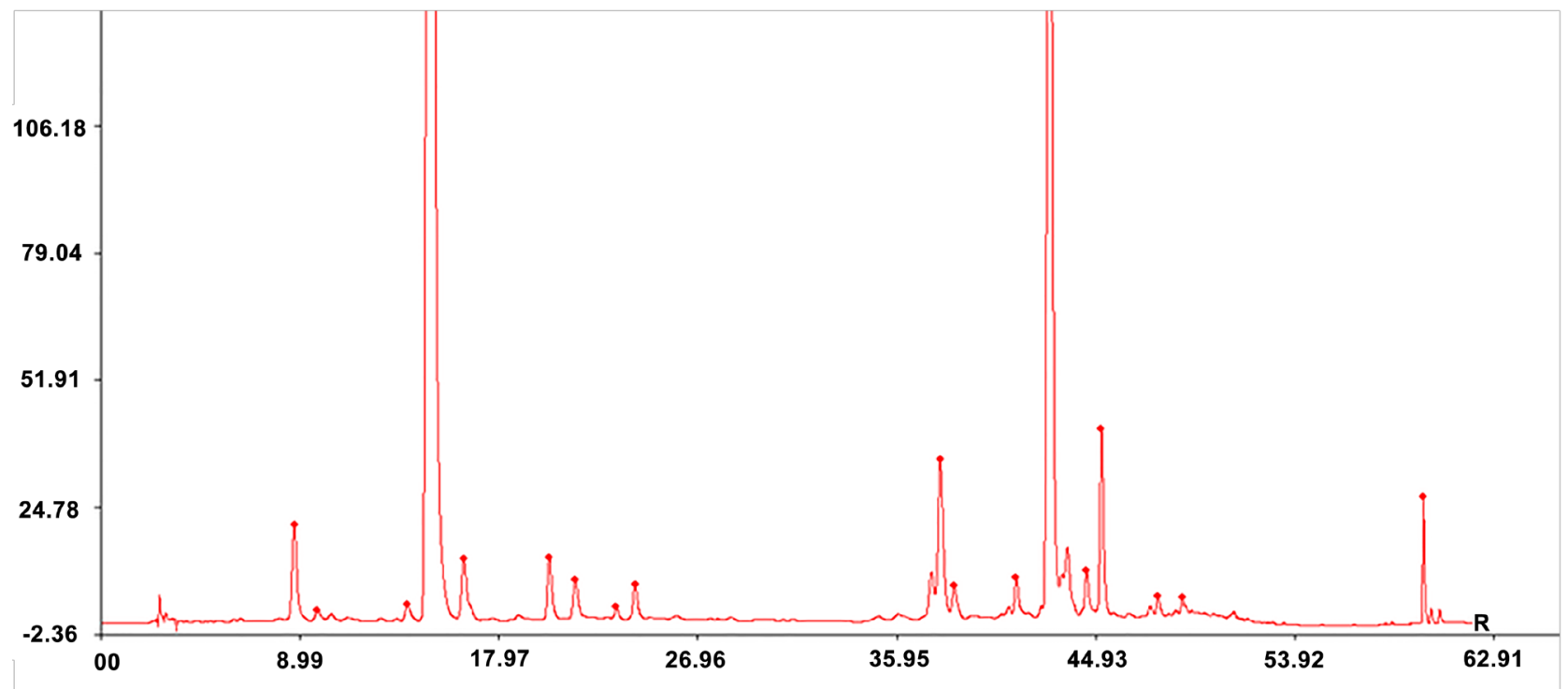

(b) Retention time (min)

Figure 8. (a) HPLC fingerprint of 11 samples of LJF from different harvest time; (b) reference chromatogram generated from all LJF samples. 
samples had generally similar spectra, indicated the similar chemical compositions of these samples. Meanwhile, the reference chromatographic fingerprint of LJF Hua Jin 6 was generated based on the 11 samples obtained from different harvest time and is shown in Figure 8(b). There were 18 common peaks (existing in all chromatograms of the 11 samples) which were marked (1 - 18) in referenced chromatographic. Peak $1(t R=8.727)$ was selected as the reference peak because it was a relatively stable peak in this chromatogram. The relative retention times (tR) and relative peak areas of these 18 common peaks are listed in Table 2.

\subsubsection{Similarity Evaluation}

It was necessary that chromatographic fingerprint of LJF from different harvest time should be evaluated by their similarities, which obtained from the calculation on the correlative coefficient of original date. The correlation coefficient between each chromatogram of LJF samples was shown in Table 3. The results indicated that the samples from different harvest time achieved a high similarity value with each other. Among of them, S2 and S9 had the lowest similarity value (0.956), and the highest similarity value achieved 1 between S1 and S2, S3 and S4, S5 and S7, S6 and S10, S8 and S11, which indicated that the total quality was stable from different harvest time. Furthermore, we calculated similarity between 11 samples and reference chromatogram, which was established through

Table 2. Relative peak areas and relative times of common peaks of LJF.

\begin{tabular}{|c|c|c|c|c|c|c|c|c|c|c|c|c|c|c|}
\hline Peak no. & $t_{R}(\min )$ & S1 & S2 & S3 & S4 & S5 & S6 & S7 & S8 & S9 & S10 & S11 & Average & RSD \\
\hline Peak 1 & 8.727 & 1 & 1 & 1 & 1 & 1 & 1 & 1 & 1 & 1 & 1 & 1 & 1 & 0.086 \\
\hline Peak 2 & 9.785 & 0.150 & 0.047 & 0.083 & 0.082 & 0.109 & 0.138 & 0.112 & 0.144 & 0.174 & 0.132 & 0.140 & 0.120 & 0.357 \\
\hline Peak 3 & 13.847 & 0.169 & 0.153 & 0.133 & 0.152 & 0.160 & 0.166 & 0.167 & 0.184 & 0.194 & 0.223 & 0.216 & 0.175 & 0.197 \\
\hline Peak 4 & 14.837 & 50.246 & 61.900 & 46.547 & 43.020 & 40.802 & 37.149 & 40.210 & 34.170 & 31.274 & 37.895 & 37.128 & 41.421 & 0.159 \\
\hline Peak 5 & 16.39 & 0.527 & 0.728 & 0.647 & 0.685 & 0.637 & 0.625 & 0.686 & 0.707 & 0.736 & 0.883 & 0.847 & 0.702 & 0.175 \\
\hline Peak 6 & 20.253 & 0.613 & 0.872 & 0.691 & 0.667 & 0.583 & 0.514 & 0.534 & 0.438 & 0.454 & 0.530 & 0.565 & 0.582 & 0.173 \\
\hline Peak 7 & 21.422 & 0.514 & 0.352 & 0.432 & 0.483 & 0.804 & 0.425 & 0.273 & 0.305 & 0.331 & 0.329 & 0.314 & 0.412 & 0.357 \\
\hline Peak 8 & 23.278 & 0.171 & 0.086 & 0.106 & 0.123 & 0.104 & 0.080 & 0.083 & 0.091 & 0.067 & 0.171 & 0.171 & 0.171 & 0.216 \\
\hline Peak 9 & 24.123 & 0.419 & 0.372 & 0.316 & 0.357 & 0.328 & 0.269 & 0.284 & 0.308 & 0.282 & 0.374 & 0.342 & 0.329 & 0.077 \\
\hline Peak 10 & 37.913 & 1.222 & 1.470 & 1.400 & 1.740 & 1.617 & 1.252 & 1.401 & 1.669 & 1.706 & 2.090 & 1.822 & 1.581 & 0.182 \\
\hline Peak 11 & 38.539 & 0.300 & 0.259 & 0.244 & 0.293 & 0.271 & 0.230 & 0.221 & 0.250 & 0.244 & 0.293 & 0.263 & 0.259 & 0.067 \\
\hline Peak 12 & 41.348 & 0.328 & 0.412 & 0.357 & 0.377 & 0.389 & 0.324 & 0.321 & 0.351 & 0.391 & 0.382 & 0.435 & 0.370 & 0.137 \\
\hline Peak 13 & 42.823 & 14.071 & 16.487 & 14.767 & 14.371 & 16.005 & 15.990 & 14.757 & 16.734 & 19.347 & 16.436 & 18.375 & 16.209 & 0.175 \\
\hline Peak 14 & 44.518 & 0.425 & 0.431 & 0.410 & 0.452 & 0.457 & 0.355 & 0.336 & 0.379 & 0.349 & 0.374 & 0.407 & 0.396 & 0.084 \\
\hline Peak 15 & 45.193 & 1.101 & 1.758 & 1.348 & 1.359 & 1.522 & 1.204 & 1.184 & 1.216 & 1.450 & 1.382 & 1.696 & 1.386 & 0.179 \\
\hline Peak 16 & 47.749 & 0.145 & 0.122 & 0.129 & 0.130 & 0.266 & 0.194 & 0.108 & 0.138 & 0.183 & 0.149 & 0.163 & 0.158 & 0.327 \\
\hline Peak 17 & 48.841 & 0.113 & 0.092 & 0.072 & 0.078 & 0.083 & 0.077 & 0.148 & 0.182 & 0.117 & 0.104 & 0.115 & 0.108 & 0.334 \\
\hline Peak 18 & 59.728 & 0.658 & 0.570 & 0.422 & 0.502 & 0.443 & 0.399 & 0.486 & 0.534 & 0.474 & 0.438 & 0.469 & 0.487 & 0.116 \\
\hline
\end{tabular}

tR is the retention time; $\mathrm{RSD}=$ Relative Standard Deviation. 
Table 3. The similarity of 11 samples investigated.

\begin{tabular}{ccccccccccccc}
\hline & S1 & S2 & S3 & S4 & S5 & S6 & S7 & S8 & S9 & S10 & S11 & R \\
\hline S1 & 1 & & & & & & & & & & \\
S2 & 1 & 1 & & & & & & & & & & \\
S3 & 0.999 & 0.999 & 1 & & & & & & & & \\
S4 & 0.999 & 0.998 & 1 & 1 & & & & & & & & \\
S5 & 0.995 & 0.993 & 0.998 & 0.999 & 1 & & & & & & \\
S6 & 0.991 & 0.989 & 0.995 & 0.996 & 0.999 & 1 & & & & & \\
S7 & 0.997 & 0.996 & 0.999 & 0.999 & 1 & 0.998 & 1 & & & & \\
S8 & 0.983 & 0.98 & 0.989 & 0.991 & 0.997 & 0.999 & 0.994 & 1 & & & & \\
S9 & 0.96 & 0.956 & 0.969 & 0.973 & 0.984 & 0.989 & 0.979 & 0.995 & 1 & & & \\
S10 & 0.99 & 0.988 & 0.994 & 0.996 & 0.999 & 1 & 0.998 & 0.999 & 0.989 & 1 & & \\
S11 & 0.982 & 0.98 & 0.988 & 0.99 & 0.996 & 0.998 & 0.994 & 1 & 0.996 & 0.999 & 1 & \\
R & 0.995 & 0.993 & 0.998 & 0.999 & 1 & 0.999 & 1 & 0.997 & 0.984 & 0.999 & 0.996 & 1 \\
\hline
\end{tabular}

mean value method. The result shown that samples from different harvest time were of high similarity with reference chromatogram, most similarity ratio is at 0.993 upwards, which further revealed that the quality of 11 samples collected from different harvest time had similar chemical compositions and this reference chromatogram could be applied as a standard HPLC fingerprint. Consequently, we think the quality of LJF was relatively stable in different harvest time in term of HPLC fingerprints.

\section{Discussion and Conclusion}

Compared to the traditional varieties of LJF, "Hua Jin 6" has a broad development prospect for its longer picking time and higher yield. However, for every Chinese herbs, there is a different optimal stage of harvesting in order to have maximum amount of bioactive compounds or yield; the results indicated that the harvest time from the first day to the eleventh day had significant impact on the amount of the active components and yield, which can make it possible to select the suitable time for different harvest purposes. Our study demonstrated that: the yield of LJF was much higher after the seventh day harvesting; the total amount of flavonoids was much higher after the eighth day harvesting; the total amount of iridoids was much higher after the fifth day harvesting; and the total amount of phenolic acids was relatively stable in all flower buds white stage. Besides, we assumed that the quality of "Hua Jin 6" is relatively stable and suitable for harvesting in all flower buds white stage in term of HPLC fingerprints.

\section{Acknowledgements}

This work was financially supported by the National Science and Technology Pillar Program during the Twelfth Five-Year Plan Period (No. 2011BAI06B01), the specialized fund for the dependent innovation of Shandong Province (2013CXC20401), Standardization construction of national TCM $\backslash(2 \mathrm{YB} 2 \mathrm{H}-\mathrm{Y}-\mathrm{SD}-32 \backslash)$. 


\section{Declaration of Interest}

The authors report no conflicts of interest.

\section{References}

[1] Han, J., Lv, Q.Y., Jin, S.Y., Zhang, T.T., Jin, S.X., Li, X.Y. and Yuan, H.L. (2014) Comparison of Anti-Bacterial Activity of Three Types of Di-O-Caffeoylquinic Acids in Lonicera japonica Flowers Based on Microcalorimetry. Chinese Journal of Natural Medicines, 12, 108-113.

[2] Zeng, Y.W., Deng, M.C., Lv, Z.C. and Peng, Y.H. (2014) Evaluation Antioxidant Activities of Extracts from 19 Chinese Edible Flowers. SpringerPlus, 3, 315. https://doi.org/10.1186/2193-1801-3-315

[3] Wang, J.H., Bose, S., Kim, G.C., Hong, S.U., Kim, J.H., Kim, J.E. and Kim, H. (2014) Flos Lonicera Ameliorates Obesity and Associated Endotoxemia in Rats through Modulation of Gut Permeability and Intestinal Microbiota. Plos ONE, 9, e86117. https://doi.org/10.1371/journal.pone.0086117

[4] Tae, J., Han, S.W., Yoo, J.Y., Kim, J.A., Kang, O.K., Baek, O.K., Lim, J.P., Kim, D.K., Kim, Y.H., Bae, K.H. and Lee, Y.M. (2003) Anti-Inflammatory Effect of Lonicera japonica in Proteinase-Activated Receptor 2-Mediated paw Edema. Clinica Chimica Acta, 330, 165-171.

[5] Li, M.Y. (2010) In Vitro Anti-Respiratory Syncytial Virus Effect of the Extraction of Lonicera japonica Thunb. Journal of Tropical Medicine, 10, 420-422.

[6] Jiang, M., Han, Y.Q., Zhou, M.G., Zhao, H.Z., Xue, X., Hou, Y.Y., Gao, J., Bai, G. and Luo, G.A. (2014) The Screening Research of Anti-inflammatory Bioactive Markers from Different Flowering Phases of Flos Lonicerae japonicae. Plos ONE, 9 , e96241. https://doi.org/10.1371/journal.pone.0096214

[7] Zhang, Y., Wang, W.Q., Guo, L.P., Rong, Q.X., Hao, Q.X., Li, W.D., Lan, J.B. and Weng, W. (2016) Study on Yield and Quality of Lonicerea Japonicae Flos in Different Harvest Seasons. Chinese Traditional and Herbal Drugs, 44, 2611-2614.

[8] Tian, L., Pu, X.F., Liu, Q., Sun, X.F., Zhang, Y.Q. and Li, J. (2015) Impact of Different Foliar Nutrition on Florescence of Lonicera japonica Thunb. Shandong Science, 28, 1-5.

[9] Wang, L.N. and Zhang, Y.Q. (2016) Characteristics and Cultivation-Outlines of Lonicera japonica New Variety Huajin 6. Journal of Anhui Agricultural Sciences, 44, 101-102.

[10] Wang, L.N., Liu, H.Y., Zhang, J., Li, J. and Zhang, Y.Q. (2014) Simultaneous Determination of Eight Bioactive Components in Lonicerae japonicae Flos by Quantitative Analysis of Multi-components by Single Marker. Chinese Journal of Experimental Traditional Medical Formulae, 20, 57-61.

[11] Liang, X.M., Jin, Y., Wang, Y.P., Jin, G.W., Fu, Q. and Xiao, Y.S. (2009) Qualitative and Quantitative Analysis in Quality Control of Traditional Chinese Medicines. Journal of Chromatography A, 1216, 2033-2044.

[12] Han, L., Liu, X.H., Yang, N.Y., Li, J.S., Cai, B.C. and Cheng, S. (2012) Simultaneous Chromatographic Fingerprinting and Quantitative Analysis of Flavonoids in Pollen Typhae by High-Performance Capillary Electrophoresis. Acta Pharmaceutica Sinica $B, 2,602-609$.

[13] Yu, K., Gong, Y.F., Lin, Z.Y. and Cheng, Y.Y. (2007) Quantitative Analysis and Chromatographic Fingerprinting for the Quality Evaluation of Scutellaria baicalensis Georgi Using Capillary Electrophoresis. Journal of Pharmaceutical and Biomed- 
ical Analysis, 43, 540-548.

[14] Chen, C., Zhang, H., Xiao, W., Yong, Z.P. and Bai, N. (2007) High-Performance Liquid Chromatographic Fingerprint Analysis for Different Origins of Sea Buckthorn Berries. Journal of Chromatography A, 1154, 250-259.

Submit or recommend next manuscript to SCIRP and we will provide best service for you:

Accepting pre-submission inquiries through Email, Facebook, LinkedIn, Twitter, etc. A wide selection of journals (inclusive of 9 subjects, more than 200 journals)

Providing 24-hour high-quality service

User-friendly online submission system

Fair and swift peer-review system

Efficient typesetting and proofreading procedure

Display of the result of downloads and visits, as well as the number of cited articles Maximum dissemination of your research work

Submit your manuscript at: http://papersubmission.scirp.org/

Or contact cm@scirp.org 